\title{
Bilateral Post-electrocution Cataract
}

\author{
Anum Javed, Owais Arshad, Javeria Nasir, Mohammad Hanif Chatni
}

Pak J Ophthalmol 2019, Vol. 35, No. 4

See end of article for authors affiliations

\section{Correspondence to:}

Dr. Owais Arshad

Ophthalmology Department,

Patel Hospital, Karachi, Pakistan

Email: owaisarshad17@gmail.com
Trauma from electricity can be of multiple forms, ranging from mild damage, to life-threatening conditions like cardiac arrest. Ophthalmic injuries are not uncommon following electrocution. We report a case of post electrocution cataract in a 24-year old male who presented to us three years after injury. On examination his best corrected visual acuity was $1 / 60$ in the right eye and hand movement in the left eye. Slit lamp examination revealed a white, mature cataract in the left eye and a developing anterior capsular cataract in the right eye. B-scan of left eye was normal. Fundoscopic examination of right eye was normal. Left cataract surgery was done. Per-operatively, the capsulorhexis was surgically challenging due to the adherence of the cataract with the anterior capsule. The BCVA in the left eye was 6/6 postoperatively.

Key Words: Electric Injury, Cataract, Phacoemulsification.
$\mathrm{E}$ lectricity related injuries are more common in developing countries like Pakistan as compared to the developed world ${ }^{1}$. Effects of electric current entering the body are a result of inflow of charges on the molecular and sub-molecular level, generating thermal energy causing disturbance of cellular physiology.

The consequences may involve any part of the body and the eyes are no exception. Cataract induced by electric current may present much later with respect to the time of injury, with rapid progression and diminishing vision ${ }^{2}$. We report a case of postelectrocution cataract in a young male.

\section{CASE REPORT}

A 24-year old male presented to the outpatient eye department of Patel hospital with complaint of decreased vision in both eyes for the past five months. His symptoms were more in the left eye. On enquiring the detailed past history he revealed that he was electrocuted 3 years ago from high tension wire affecting the left side of his body. On physical examination, there was a wound of electrical injury on the left palm. BCVA in the right eye was $1 / 60$, and Hand Movement in the left eye. Slit lamp examination showed a white, mature cataract with adherent central plaque to the anterior capsule in the left eye as shown in Figure 1 (left side). In the right eye, there was also a developing anterior sub capsular plaque like cataract similar to the left eye (Right side of figure 1).

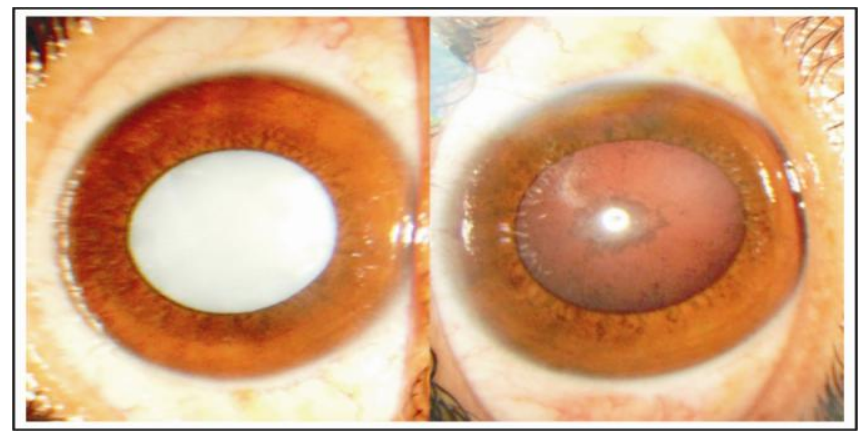

Fig. 1 (Left): White, mature cataract in the left eye, adherent to the anterior capsule.

(Right): Cataract developing in the right eye.

Dilated fundus examination of right eye was normal. B-Scan ultrasound was done which showed a flat retina. Left cataract surgery was planned. During phacoemulsification, the anterior capsule was adherent with the underlying cortex forming a plaque. Capsulorhexis was extended towards the zonules and 
typical Argentinian flag sign was observed but the situation was managed and acrylic soft hydrophobic posterior chamber intra-ocular lens (PC-IOL) was implanted in the bag under general anesthesia. Postoperatively, unaided vision in the left eye was $6 / 6$ with normal fundoscopic findings on 2-weekly followup. Right eye surgery was also planned in the following month. Informed consent was obtained from the patient for reporting this case.

\section{DISCUSSION}

Ocular post electrocution injuries include entities like cystic macular edema/macular cyst, iritis, anisocoria, chemosis and corneo-epithelial keratitis. Among these, lens is particularly more vulnerable to electric current. Therefore, cataract remains the commonest form of electrical ocular injury. There are several factors, which are responsible for the variable manifestations and features of these pathologies including location and orientation of body tissues in the current path, duration of electric current as well as its amperage, voltage and resistance ${ }^{3}$.

Etiology of electric cataract is unknown but thought to be related to the protein coagulation in the lens that occurs after an electric shock. Worldwide, post-electrocution cataract occurs in $0.8 \%$ of the population $^{4}$. Even there is high prevalence of electrocution cases in our country, but due to lack of awareness and increased mortality, very few cases have been reported.

Duration in which the visually significant cataract develops ranges from 1-18 months ${ }^{5}$ as in our case, the patient presented after three years of latent period following injury. Patient usually remains asymptomatic for a long duration with subsequent decreasing vision over a short period of time, as cataract approaches the visual axis, like in our case.

Morphologically the cataract starts as a vacuolar appearance in the mid-periphery of the lens which may be easily missed out in an undilated examination ${ }^{6}$. Cataract in these cases, may have a strong attachment anteriorly and/or posteriorly with the capsule, making it a surgical challenge ${ }^{7}$. In our case also, due to the adherent nature of the cataract to the capsule, it was forming a plaque like appearance with cortical spokes. Surgically, it is possible to extract this cataract via phacoemulsification with PC-IOL, provided it is performed by a well-trained surgeon owing to the peculiar nature of this type of cataract. In our case, the Capsulorhexis was difficult. The capsule was unidentifiable as a separate entity due to its rubbery and adherent nature as found in other few cases that are reported. PC-IOL is usually implanted after extraction of the cataract provided that the capsule is intact. Post operatively, the visual outcome is good $^{5}$, in most of the cases as in our case.

\section{CONCLUSION}

Post electrocution cataract may present late. Detailed history is important in finding out the cause of presenile cataract. Cataract surgery of such patients may be surgically challenging.

\section{GRANT SUPPORT \& FINANCIAL DISCLOSURES}

None.

\section{REFERENCES}

1. Zeb A, Arsh A, Bahadur S, Ilyas SM. Spinal cord injury due to fall from electricity poles after electrocution. Pak J Med Sci. 2019; 35 (4): 1036-1039.

2. Zhang L, Zhang K, Zhu YN, Wang QW, Yao K. Case report of unilateral electric cataract with transmission electron microscopy image. Int J Ophthalmol. 2016; 9 (4): 636-637.

3. Fish RM, Geddes LA. Conduction of electrical current to and through the human body: a review. Eplasty. 2009; 9: e44.

4. Kasana RA, Baba PU, Wani AH. Pattern of high voltage electrical injuries in the Kashmir valley: a 10-year single centre experience. Ann Burns Fire Disasters, 2016; 29 (4): 259-263.

5. Baranwal VK, Satyabala K, Gaur S, Dutta AK. A case of electric cataract. Med J Armed Forces India, 2014; 70 (3): 284-285.

6. Sofi R, Qureshi T, Gupta V. Electric cataracts: a cause of bilateral blindness in Kashmir. Eye (Lond). 2018; 32 (10): 1676-1677.

7. Kumawat D, Ramananda K, Sahay $\mathbf{P}$, et al. Posterior capsular rupture and spontaneous posterior dislocation of lens following electrical injury. Case Reports, 2017; 2017: bcr-2017-222765. 


\section{Author's Affiliation}

Dr. Anum Javed

Department of Ophthalmology,

Patel Hospital, Karachi, Pakistan.

Dr. Owais Arshad

Department of Ophthalmology,

Patel Hospital, Karachi, Pakistan

Dr. Javeria Nasir

Department of Ophthalmology,

Patel Hospital, Karachi, Pakistan

Dr. Mohammad Hanif Chatni

Department of Ophthalmology,

Patel Hospital, Karachi, Pakistan

\section{Author's Contribution}

Dr. Anum Javed

Manuscript Design, Writing and final review.

Dr. Owais Arshad

Manuscript Design and critical review.

Dr. Javeria Nasir

Manuscript Design and Critical review.

Dr. Mohammad Hanif Chatni

Manuscript Design and Critical review 